\title{
Supporting Information for Stepwise On-Surface Synthesis of Porous Carbon Nanoribbons with Notched Zigzag Edges
}

Suqin Cheng, ${ }^{\dagger}$ Xuelei Su, ${ }^{\dagger}$ Fuwei Gan,${ }^{\ddagger}$ Chengshuo Shen, ${ }^{\ddagger}$ Huibin Qiu, ${ }^{*}{ }^{\ddagger}$ Kun Tao, ${ }^{*}$, and Ping $\mathrm{Yu}^{*, \dagger}$

$\dagger$ School of Physical Science and Technology, ShanghaiTech University, 201210 Shanghai, China

$\ddagger$ School of Chemistry and Chemical Engineering, state Key Lab of Metal Matrix

Composites, Shanghai Jiao Tong University, 200240 Shanghai, China

\Key Lab for Magnetism and Magnetic Materials of Ministry of Education, Lanzhou University, 730000 LanZhou, China

E-mail: hbqiu@sjtu.edu.cn; taokun@lzu.edu.cn; yuping@shanghaitech.edu.cn

\section{Content:}

- Synthesis of U-shaped monomer precursors

- Molecular model of a typical self assembled precursors at room temperature

- Statistics on the yield of nanoporous structrues at different preparation temperatures

- $\mathrm{d} I / \mathrm{d} V$ mappings and DFT calculation results of two and three rows of P-Z-ribbon heterostructures

- Clar's theory analysis 


\section{Synthesis of U-shaped monomer precursors}

Most experiments were performed under an atmosphere of dry nitrogen using standard Schlenk techniques. Commercially available reagents were used as received without further purification. Irradiation reactions were conducted using a mercury vapor lamp (1000 W, Shanghai Qiaofeng). NMR spectra were recorded on Bruker AVANCE III HD $500 \mathrm{MHz}$ spectrometer. ${ }^{1} \mathrm{H}$ and ${ }^{13} \mathrm{C}$ chemical shifts were determined using residual signals of the deuterated solvents or using TMS as the internal standard, and the reported in parts per million (ppm) relative to TMS.

1,3-bis(bromomethyl)-2-chlorobenzene (1): A mixture of 2-chloro-1,3-dimethylbenzene (10.6 mL, $80 \mathrm{mmol}$ ), N-bromosuccinimide $(30 \mathrm{~g}, 168 \mathrm{mmol})$ and AIBN $(200 \mathrm{mg})$ in $\mathrm{CHCl}_{3}$ $(250 \mathrm{~mL})$ was heated at $60^{\circ} \mathrm{C}$ for $3 \mathrm{~h}$ under $N^{2}$ and monitored by TLC. The white solid succinimide was removed by filtration. The solvent of the filtrate was evaporated under vacuum and the resulting solid was purified by column chromatography in silica gel using heptane, affording $11 \mathrm{~g}$ of product 1,3-bis(bromomethyl)-2-chlorobenzene (1) as a white solid in $46 \%$ yield. ${ }^{1} \mathrm{H} \mathrm{NMR}\left(\mathrm{CDCl}_{3}, 500 \mathrm{MHz}, 295 \mathrm{~K}\right)$, ppm: $4.61(\mathrm{~s}, 4 \mathrm{H}), 7.24(\mathrm{~d}, \mathrm{~J}=7.6 \mathrm{~Hz}$, $2 \mathrm{H}), 7.42(\mathrm{t}, \mathrm{J}=7.6 \mathrm{~Hz}, 1 \mathrm{H})$.
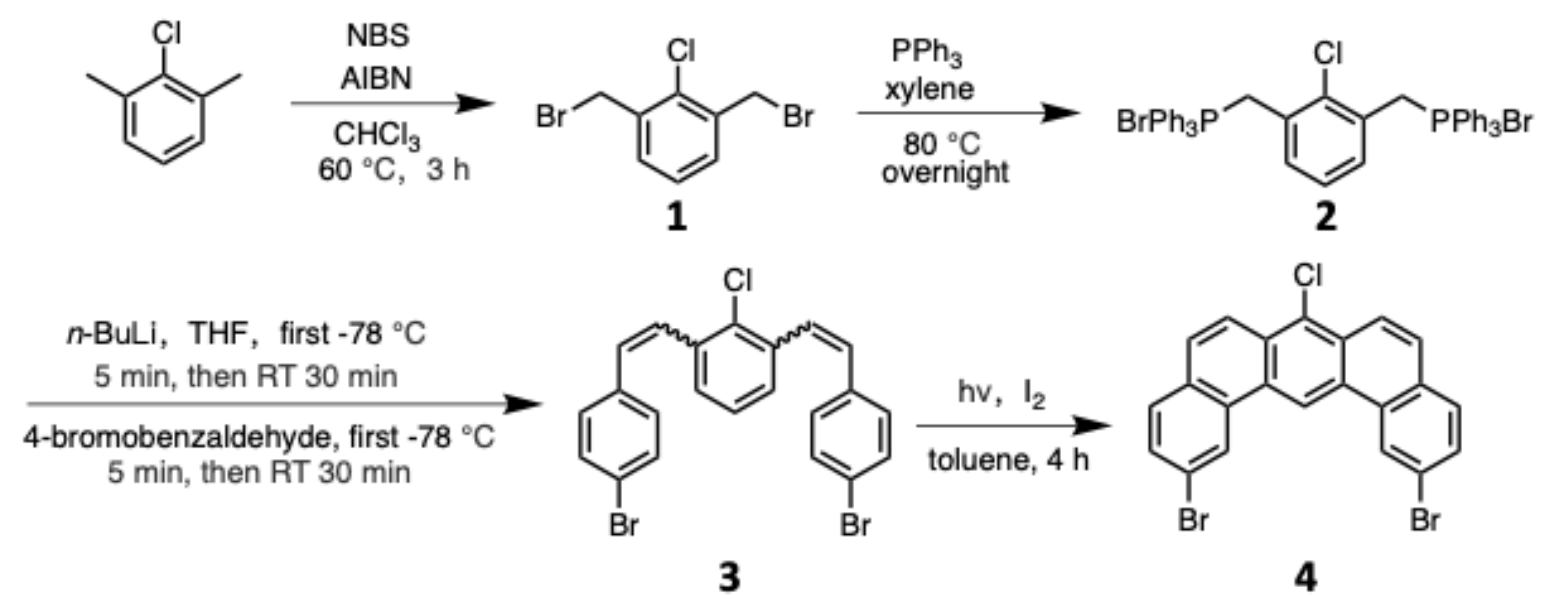

Figure S1: reaction steps for synthesis U-shaped precursors

1,3-bis((triphenylphosphono)methyl)-2-chlorobenzene bromide (2): A solution of 1,3- 


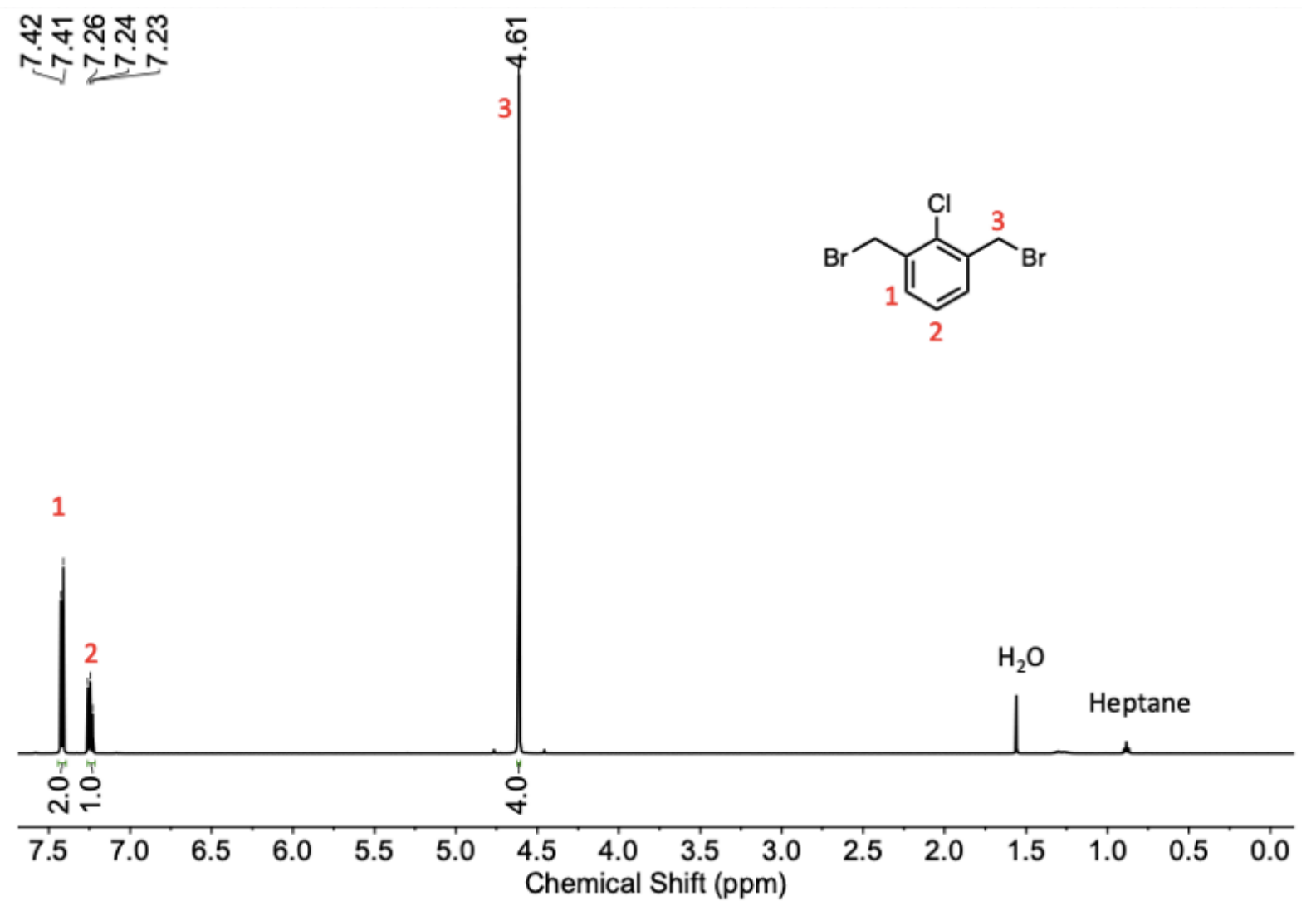

Figure S2: ${ }^{1} \mathrm{H}$ NMR spectrum of compound $1\left(500 \mathrm{MHz}, C D C l_{3}, 295 \mathrm{~K}\right)$ 
N

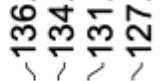

p

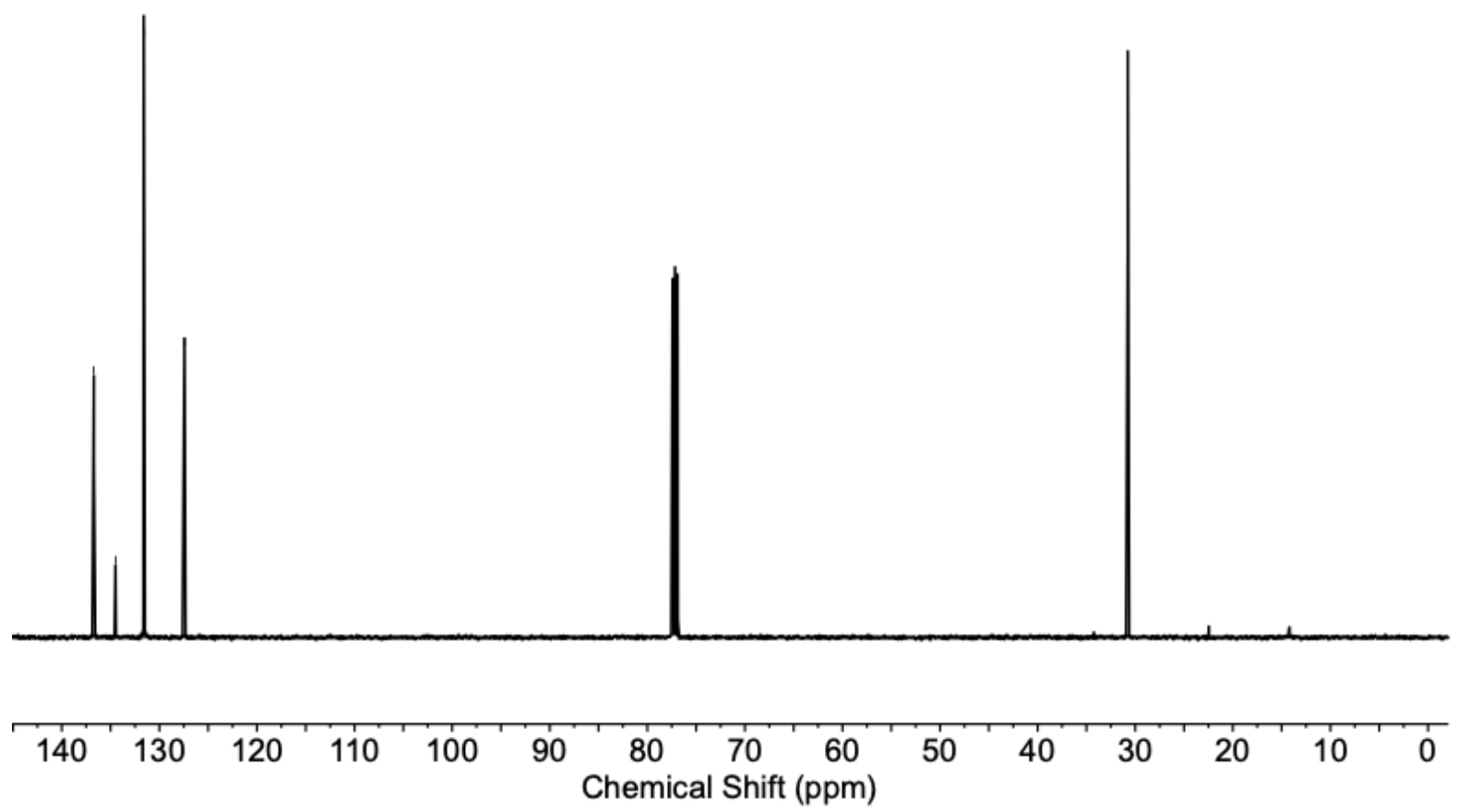

Figure S3: ${ }^{13} C$ NMR spectrum of compound $1\left(500 \mathrm{MHz}, C D C l_{3}, 295 \mathrm{~K}\right)$ 


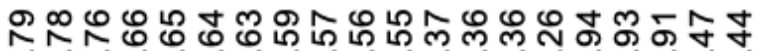

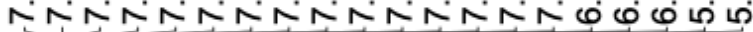

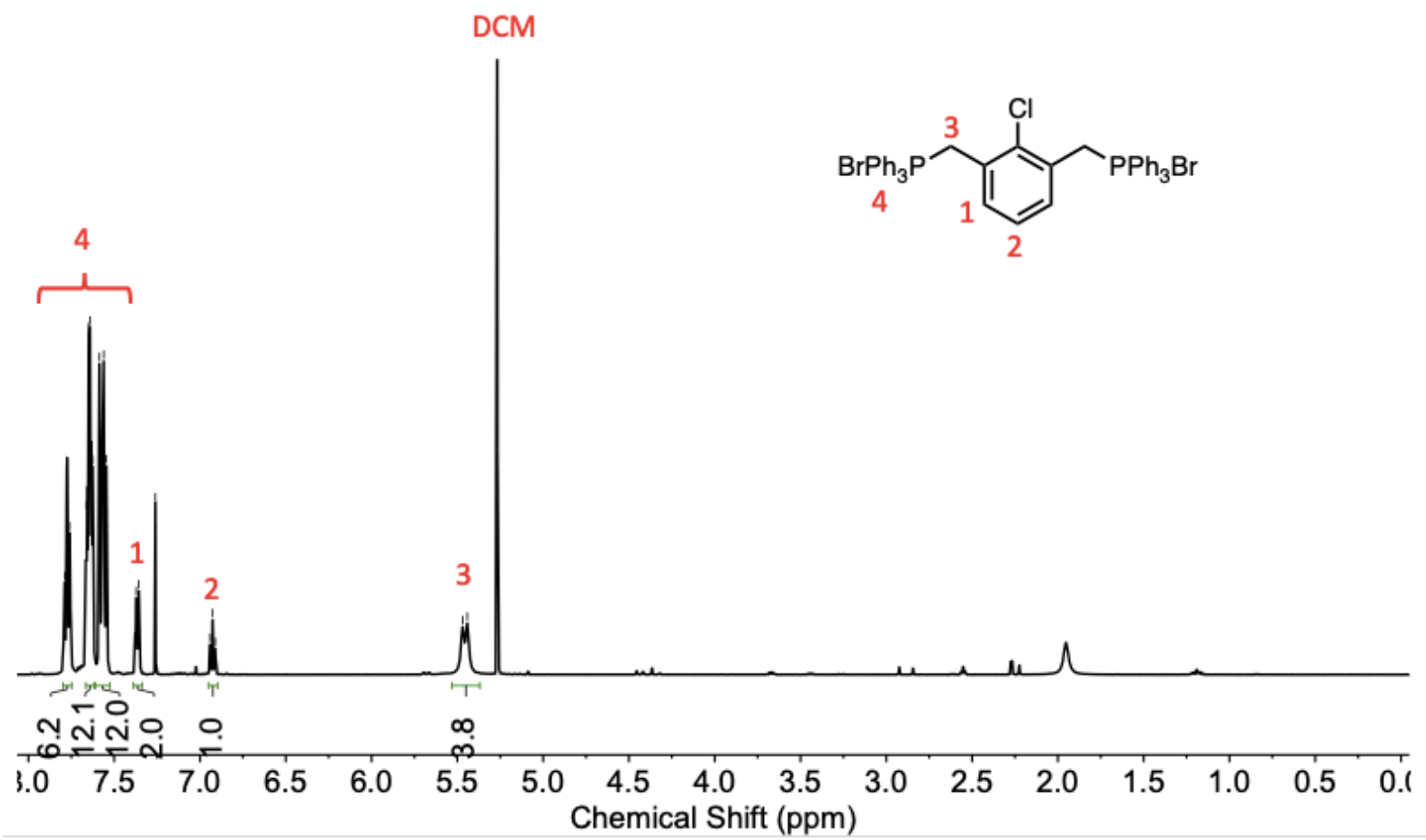

Figure S4: ${ }^{1} \mathrm{H}$ NMR spectrum of compound $2\left(500 \mathrm{MHz}, C D C l_{3}, 295 \mathrm{~K}\right)$ 


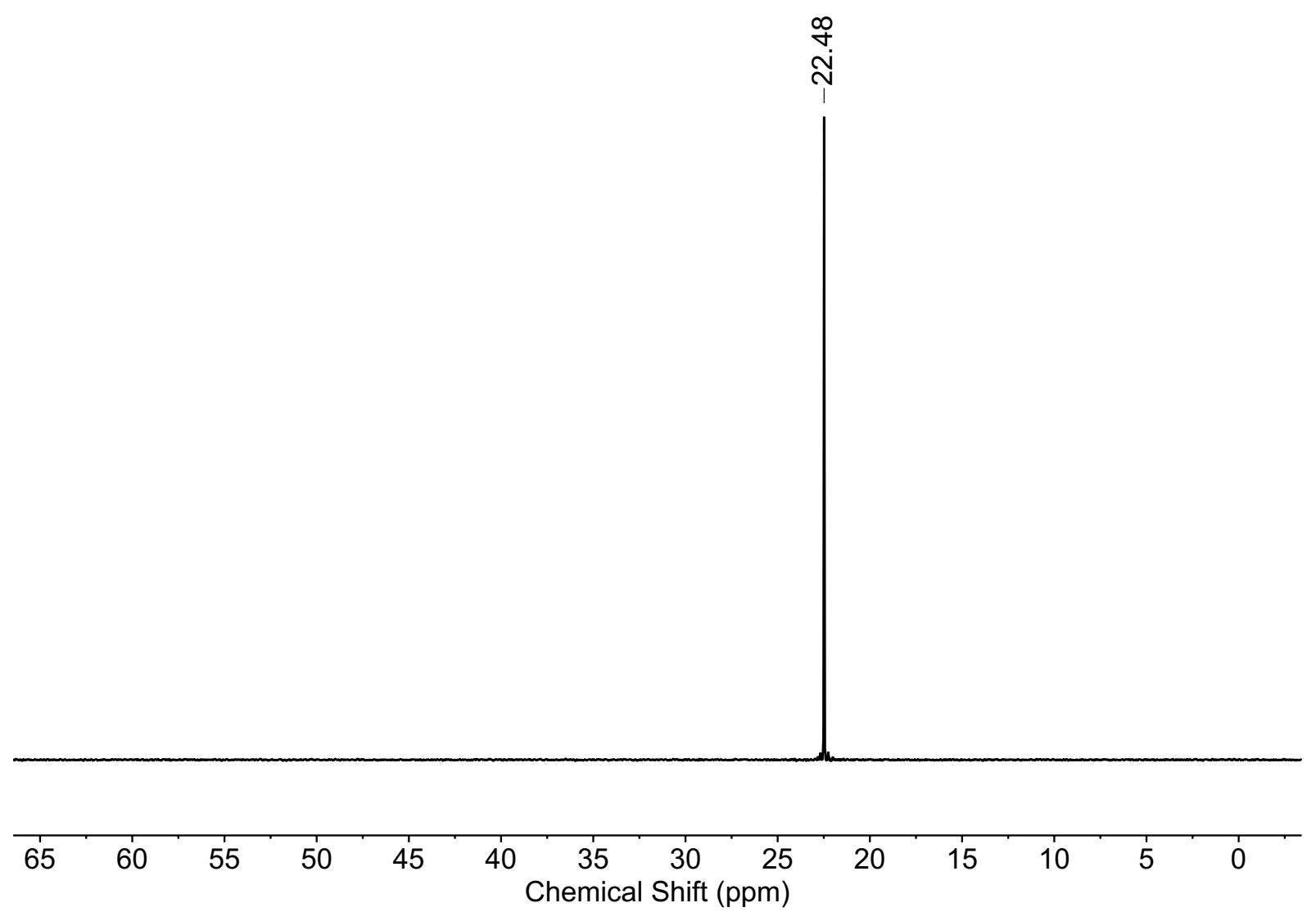

Figure S5: ${ }^{31} \mathrm{P}$ NMR spectrum of compound $2\left(500 \mathrm{MHz}, C D C l_{3}, 295 \mathrm{~K}\right)$ 


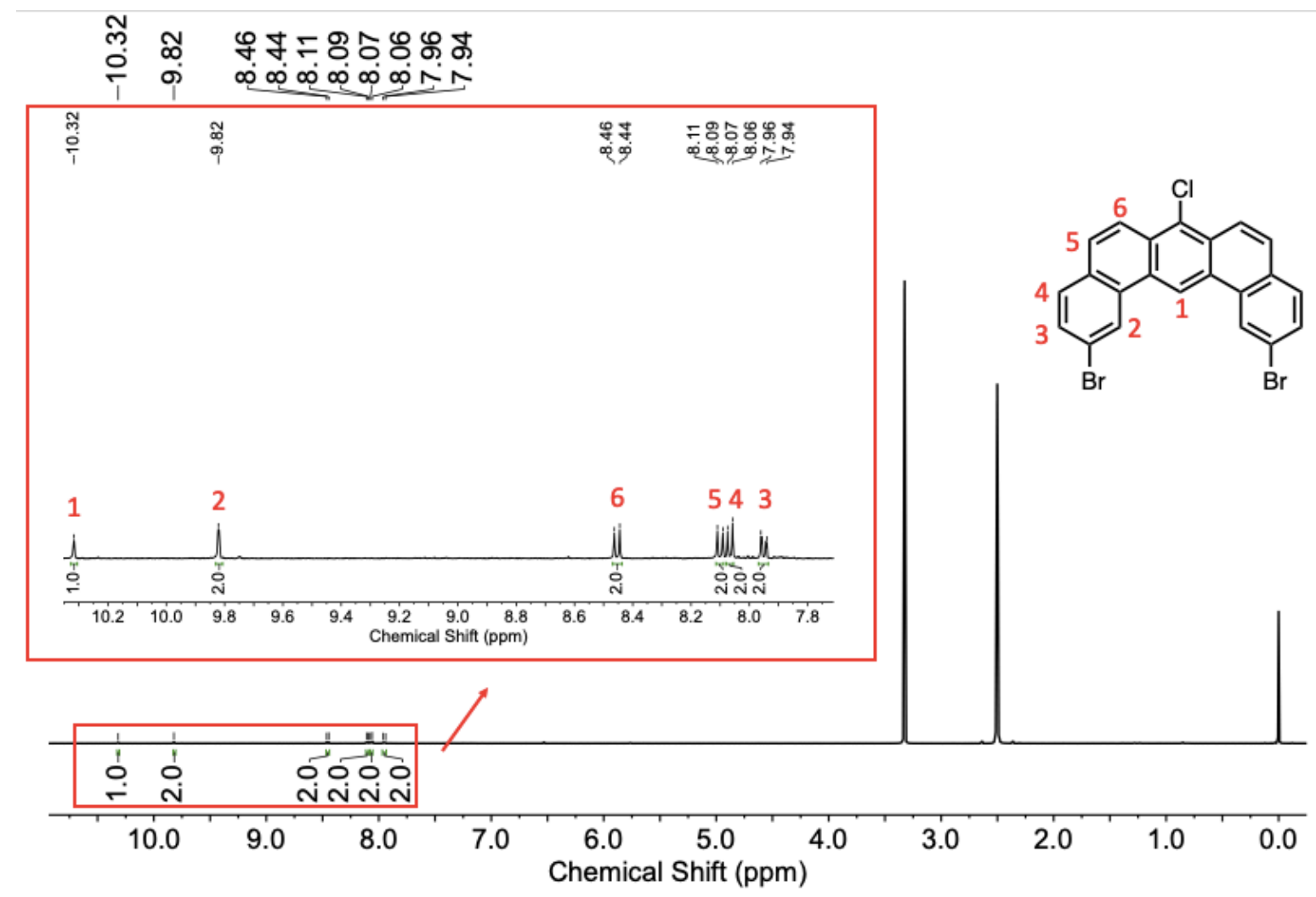

Figure S6: ${ }^{1} H$ NMR spectrum of compound $4\left(500 \mathrm{MHz}, D M S O-d_{6}, 295 \mathrm{~K}\right)$

bis(bromomethyl)-2-chlorobenzene (10.4 g, $35 \mathrm{mmol})$ and PPh3 (20 g, $76 \mathrm{mmol})$ in $500 \mathrm{~mL}$ of xylene was heated at $80^{\circ} \mathrm{C}$ overnight. The product 1,3-bis((triphenylphosphono)methyl)2-chlorobenzene bromide (2) as a white solid was obtained by filtration. ${ }^{1} \mathrm{H}$ NMR $\left(C D C l_{3}\right.$, $500 \mathrm{MHz}, 295 \mathrm{~K})$, ppm: $5.45(\mathrm{~d}, \mathrm{~J}=13.44 \mathrm{~Hz}, 4 \mathrm{H}), 6.93(\mathrm{t}, \mathrm{J}=7.76 \mathrm{~Hz}, 1 \mathrm{H}), 7.36(\mathrm{dt}, \mathrm{J}$ $=7.8 \mathrm{~Hz}, 2.53 \mathrm{~Hz}, 2 \mathrm{H}), 7.59-7.82(\mathrm{~m}, 30 \mathrm{H})$.

2,12-dibromo-7chlorobenzo[m]tetraphene (4): 1,3-bis((triphenylphosphono)methyl)-2-chlorobenzene bromide ( $8.64 \mathrm{~g}, 10 \mathrm{mmol}$ ) was suspended in $100 \mathrm{~mL}$ of dry THF under nitrogen and cooled to $-78^{\circ} \mathrm{C} .13 .8 \mathrm{~mL}$ of n-butyllithium (1.6 molL-1 in hexane, $22 \mathrm{mmol}, 2.2$ equiv.) was added, and the reaction mixture was stirred at $-78^{\circ} \mathrm{C}$ for $5 \mathrm{~min}$ and then at room temperature for $30 \mathrm{~min}$. The reaction mixture turned red during the procedure. Then the reaction mixture was cooled to $-78^{\circ} \mathrm{C}$ again and 4-bromobenzaldehyde (4 g, $\left.21 \mathrm{mmol}\right)$ dissolved in dry THF was added dropwise into the solution. The reaction was stirred at $-78^{\circ} \mathrm{C}$ for $5 \mathrm{~min}$ and then at room temperature for $2 \mathrm{~h}$. The solvent was removed in vacuum and the product 


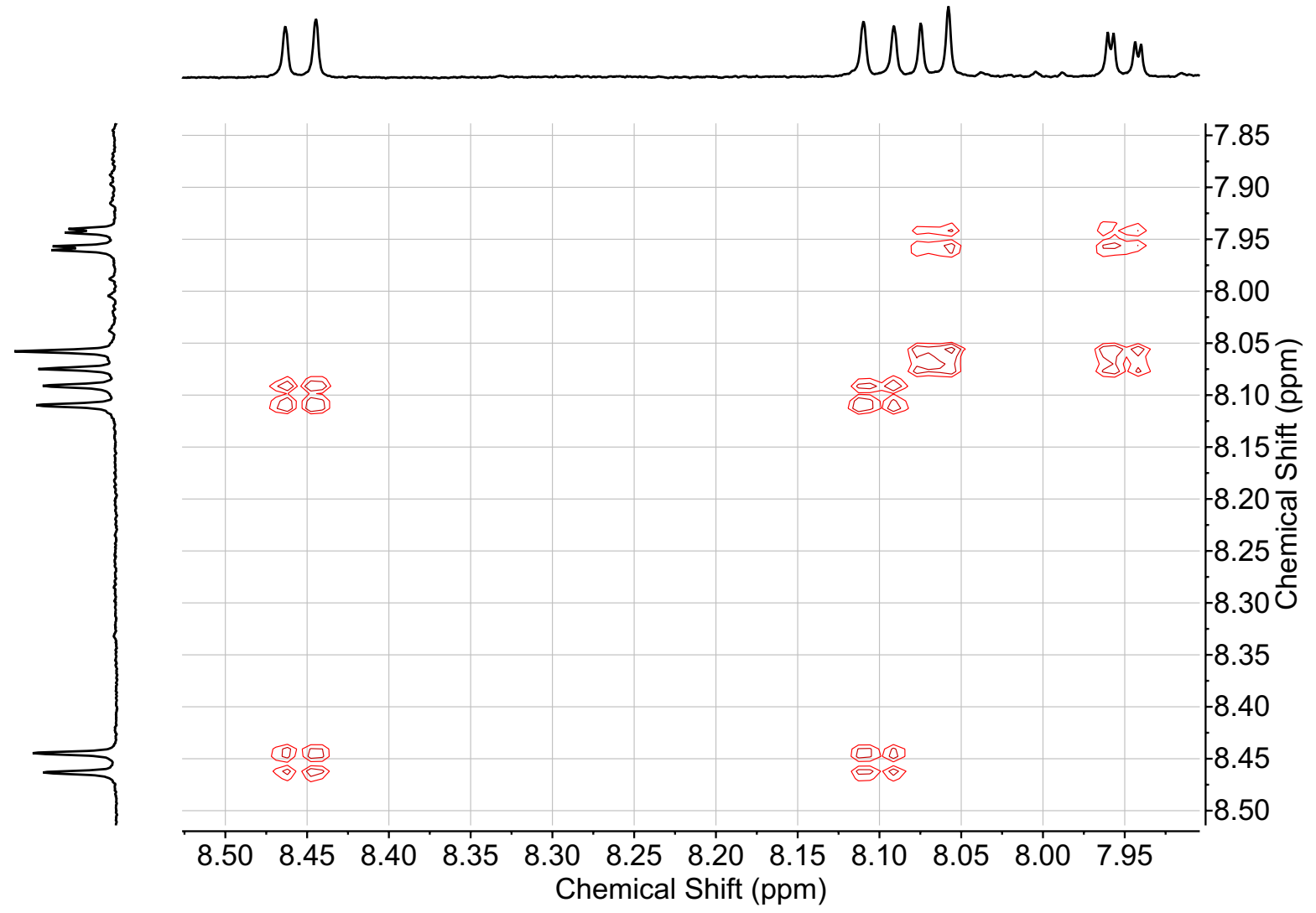

Figure S7: ${ }^{1} H-{ }^{1} H$ COSY spectrum of compound $4\left(500 \mathrm{MHz}, D M S O-d_{6}, 295 \mathrm{~K}\right)$ 
was purified by short silica column chromatography (eluent: dichloromethane) to afford the stilbene derivative (3) as yellow solid (3.56 g, yield 75\%, mixture of cis and trans). Then this stilbene derivative was dissolved in toluene $(10 \mathrm{~L})$ together with $5 \mathrm{~g}$ I2. The solution was divided to five parts and irradiated under mercury lamp for $4 \mathrm{~h}$ respectively. Then the solution was removed and the residue was washed by dichloromethane to afford the product 2,12-dibromo-7-chlorobenzo[m]tetraphene (4) as paled yellow solid (490 mg, yield 14\% ). $46 \%$ NMR $\left(D M S O-d_{6}, 500 \mathrm{MHz}, 295 \mathrm{~K}\right), \operatorname{ppm}: 7.95(\mathrm{~d}, \mathrm{~J}=9.47 \mathrm{~Hz}, 2 \mathrm{H}), 8.07(\mathrm{~d}, \mathrm{~J}=$ $8.58 \mathrm{~Hz}, 2 \mathrm{H}), 8.11(\mathrm{~d}, \mathrm{~J}=9.29 \mathrm{~Hz}, 2 \mathrm{H}), 8.43(\mathrm{~d}, \mathrm{~J}=9.2 \mathrm{~Hz}, 2 \mathrm{H}), 9.81(\mathrm{~s}, 2 \mathrm{H}), 10.27(\mathrm{~s}, 1 \mathrm{H})$.

Please note that the ${ }^{13} \mathrm{C}$ NMR spectrum is not shown for compound 4 is due to its poor solubility. With trying several different solvents, it can only be dissolved a little bit in dimethy sulfoxide (DMSO) which is still not sufficient for ${ }^{13} \mathrm{C}$ NMR and high resolution mass spectrum measurements. But from the STM/AFM experiments, the molecular structure of compound 4 should be confirmed.

\section{Molecular model of a typical self assembled precursors at room temperature}

At room temperature, the U-shaped precursors mostly form the self-assembled structures with the type I and type II halogen bonds interaction. ${ }^{1}$

\section{Statistics on the yield of nanoporous structures at different prepa- ration temperatures}

The annealing temperature at the second reaction step is set as $673 \mathrm{~K}$, which is high enough for both $\mathrm{Cl}$ dissociation and the dehydrogenation to avoid the hydrogen steric blocking

in the peri positions. For evidencing the hydrogen steric blocking influence on the yield of nanoporous structures, $593 \mathrm{~K}$ as the second reaction temperature has been tried for comparison, which temperature can only trigger the $\mathrm{Cl}$ dissociation. As the Fig.S8a shown, 


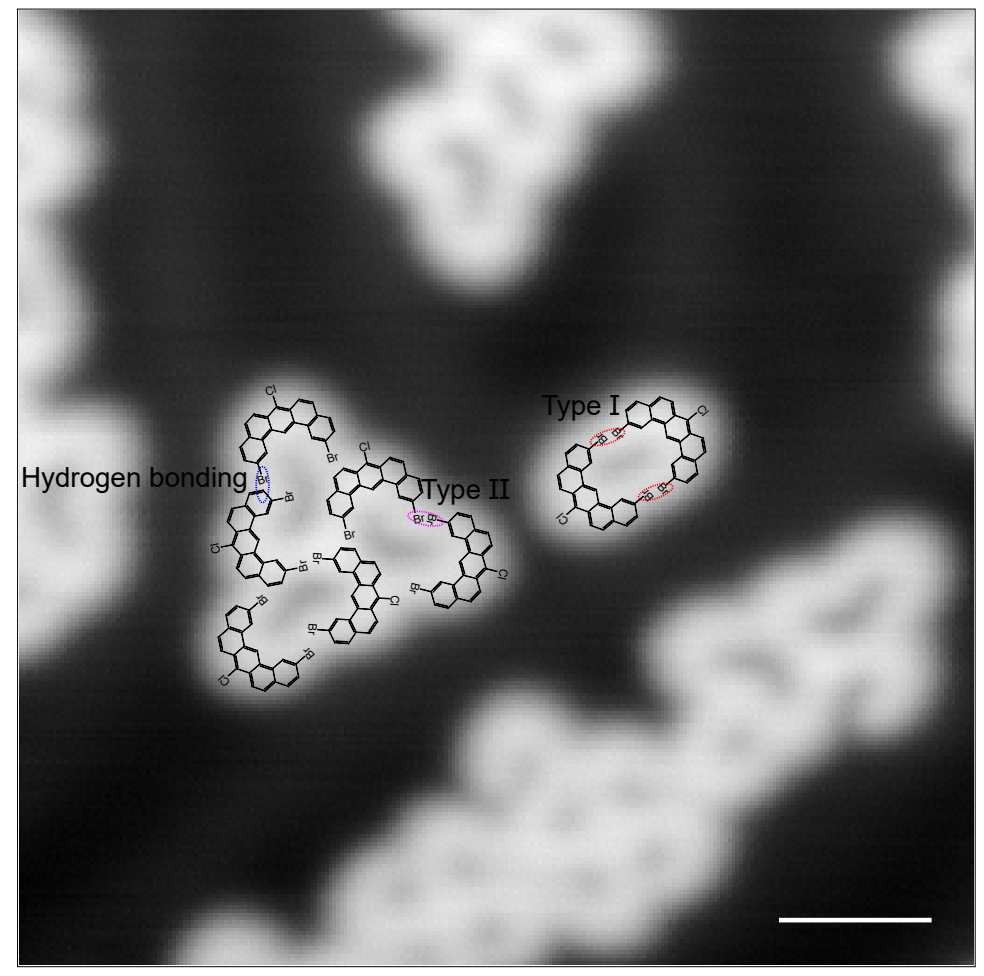

Figure S8: molecular model for self-assembled precursors at room temperature. Scale bar, $2 \mathrm{~nm}$ 
the ratio of the number of precursors forming nanoporous ribbons to the total number of precursors annealing at $593 \mathrm{~K}$ is only 3 percent, while the ratio for annealing at $673 \mathrm{~K}$ is increased up to 16 percent (about 2000 precursor molecules are counted for statistics for each annealing temperature). Moreover, for the analysis on the length distribution of the nanoporous ribbons forming at these two temperatures, about 70 percent of the nanoporous ribbons forming at $593 \mathrm{~K}$ have the length below $4 \mathrm{~nm}$. In contrast, the lengths of nealy 60 percent of the nanoporous ribbons forming at $673 \mathrm{~K}$ are above $4 \mathrm{~nm}$, which both suggest that by triggering the $\mathrm{Cl}$ dissociation and dehydrogenation simultaneously can effectively reduce the hydrogen steric blocking in the peri positions and promote the yield of nanoporous structures.

\section{$\mathrm{d} I / \mathrm{d} V$ mappings and DFT calculation results of two and three rows of P-Z-ribbon heterostructures}

For the two and three rows of P-Z-ribbon heterostructures, the $\mathrm{d} I / \mathrm{d} V$ mappings of three typical electronics states are shown in the insets of Fig. S8c,d, which show the similar intensity distribution as that of one row of P-Z-ribbon. In comparison with the calculation results shown in Fig.S8e,d, the intensity distribution of the electronic states agrees well between theoretical and experimental results. The obvious difference between calculation and experiment results is that, the muti-ribbon correlation effect is clearly reflected in DFT calculation results that the first positive and negative electronic states appearing as double and triple peaks for two and three rows of P-Z-ribbon heterostructures, while only one electronic state can be clearly detected in experiments for both two and three rows. The discrepency could be due to the strong convelution of the elelctronic states which is beyond our $\mathrm{d} I / \mathrm{d} V$ spectra energy resolution. While in experimental $\mathrm{d} I / \mathrm{d} V$ spectra, the first negative electronic states are obviously getting broader and weaker. 
a

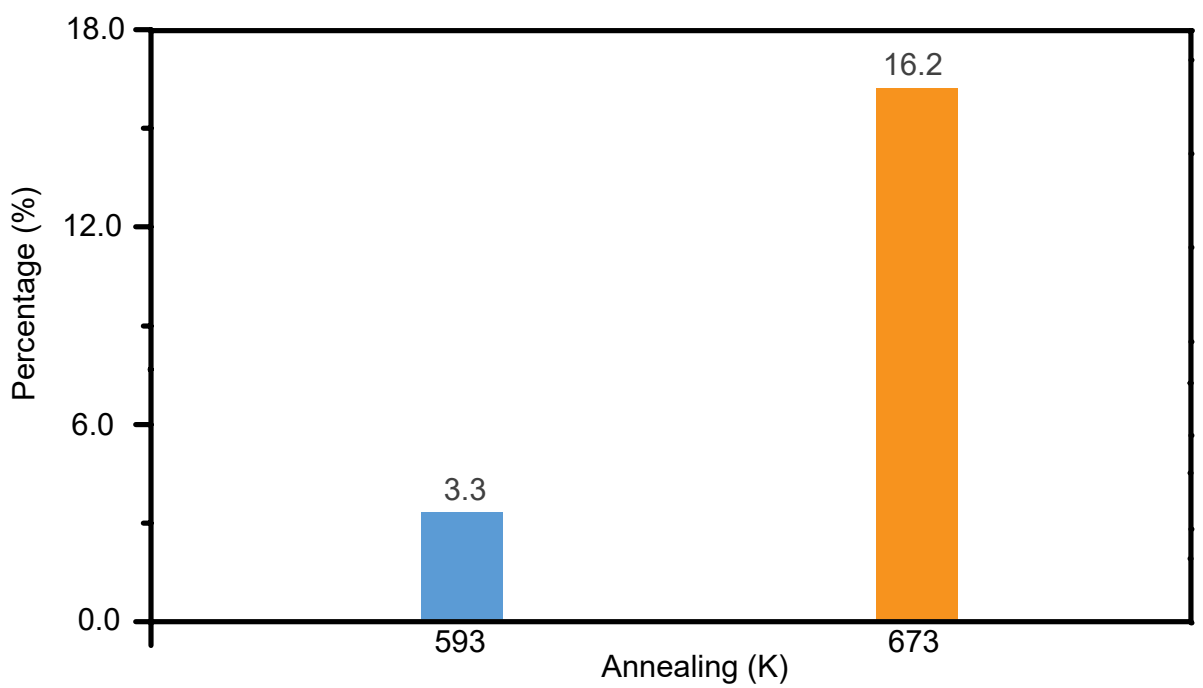

b

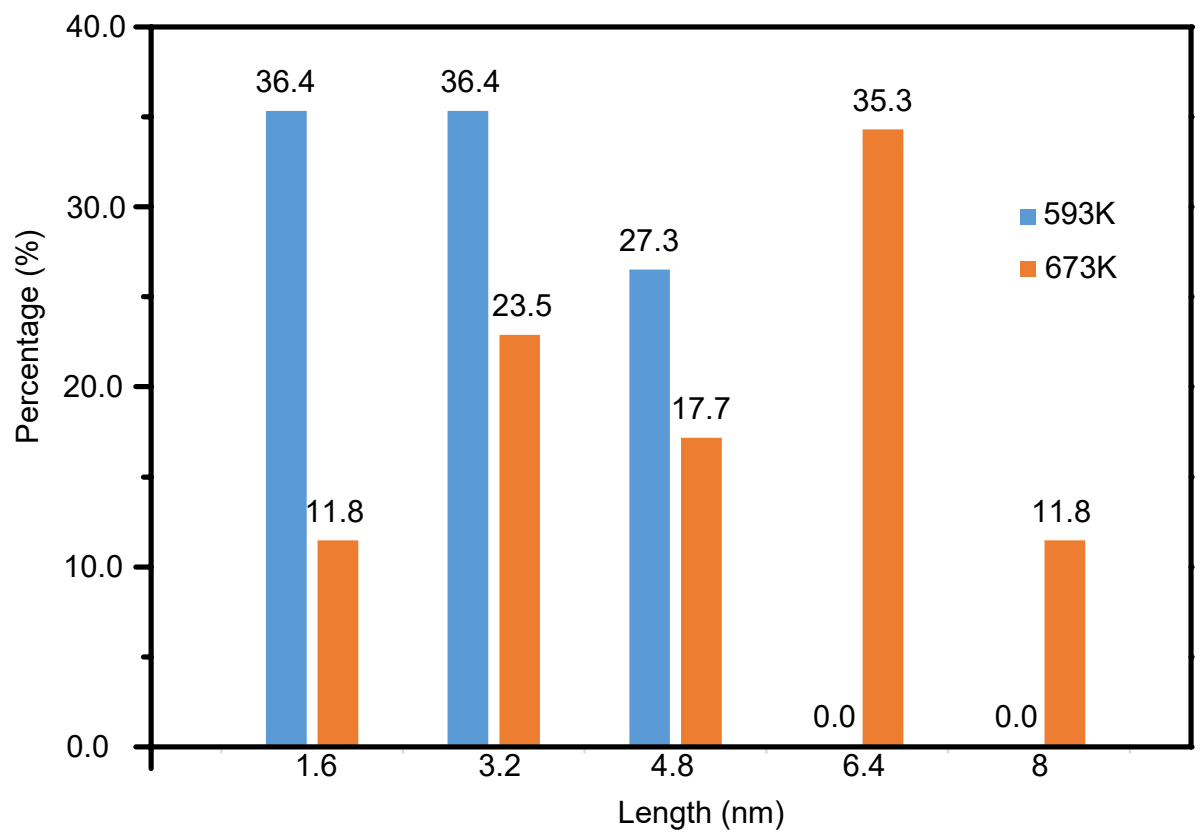

Figure S9: (a) The statistics on the yield of nanoporous structures at different preparation temperatures (b) The length distribution of the nanoporous structures forming at different preparation temperatures. 
a

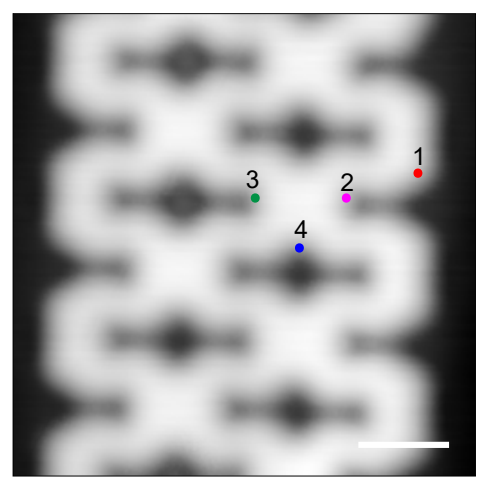

c

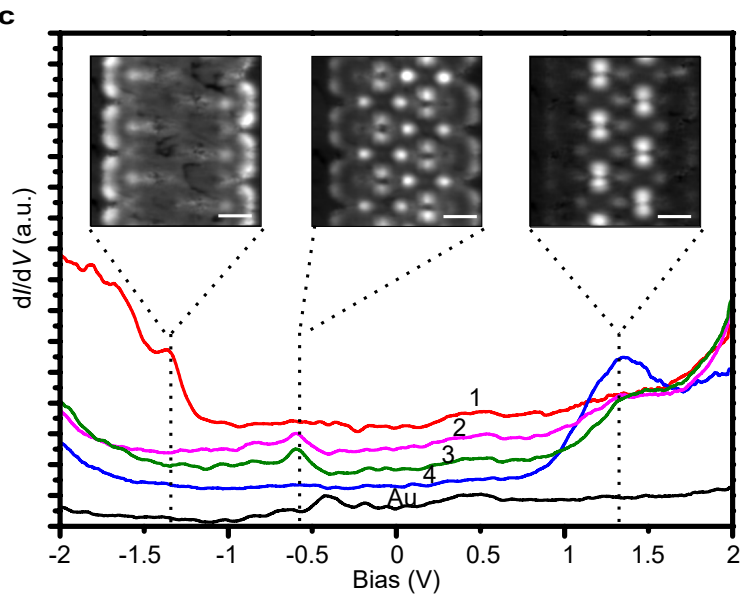

e

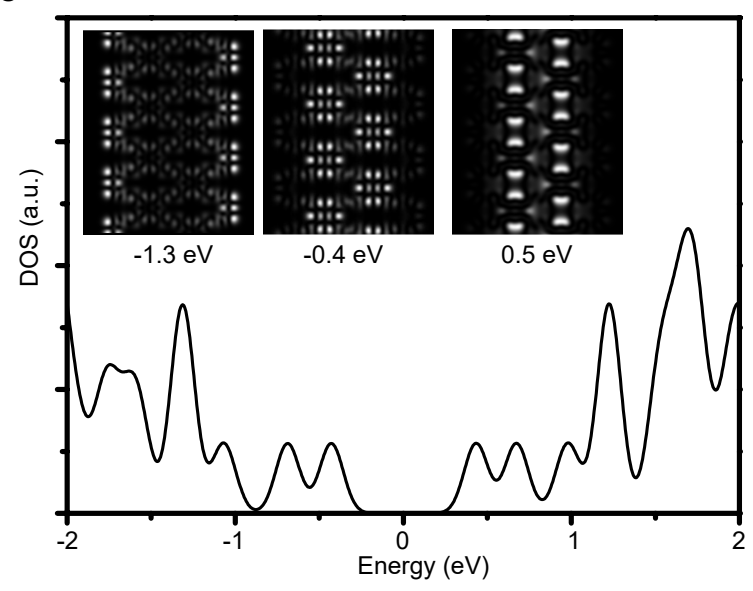

b

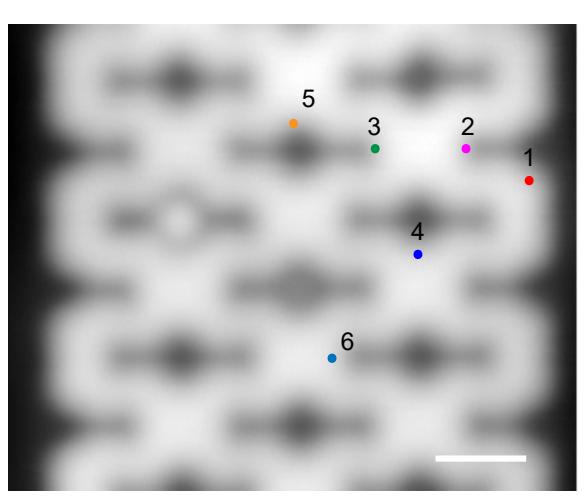

d

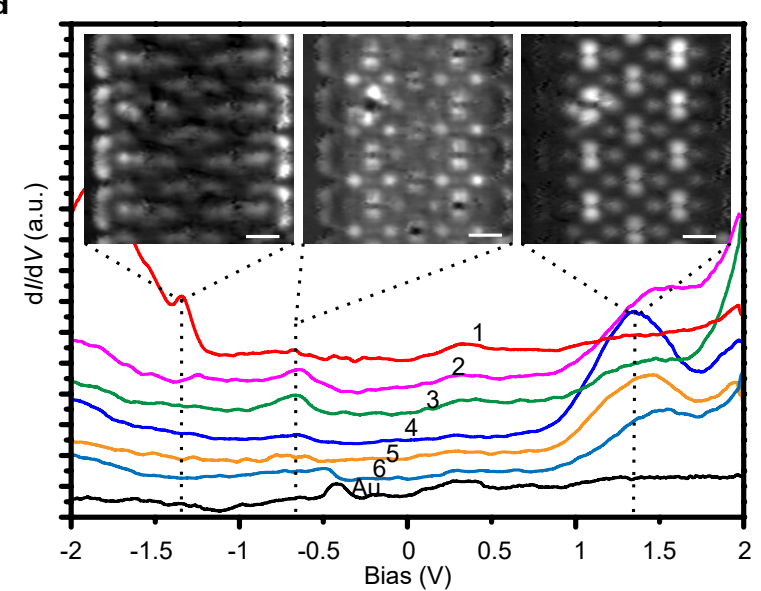

f

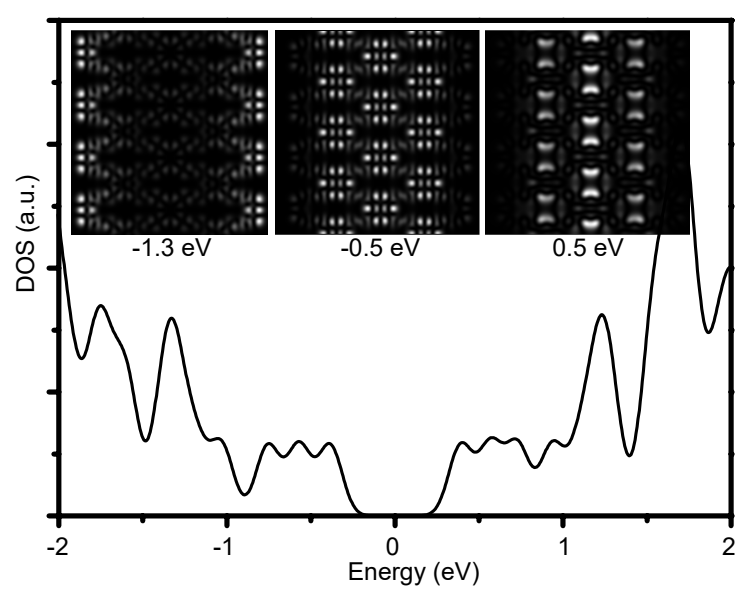

Figure S10: $(a, b)$ The STM images of two and three rows of P-Z-ribbon heterostructures. $(\mathrm{c}, \mathrm{d})$ The $\mathrm{d} I / \mathrm{d} V$ spectra measured at specific positions as marked in $(\mathrm{a}, \mathrm{b})$ and the corresponding $\mathrm{d} I / \mathrm{d} V$ mappings taken at $-1.35 \mathrm{eV},-0.65 \mathrm{eV}$ and $1.3 \mathrm{eV}$. (e,f) DFT calculation results of the $\mathrm{d} I / \mathrm{d} V$ spectra and the $\mathrm{d} I / \mathrm{d} V$ mappings for two and three rows of P-Z-ribbon heterostructures. Scale bars, $1 \mathrm{~nm}$ 
$\mathbf{a}$

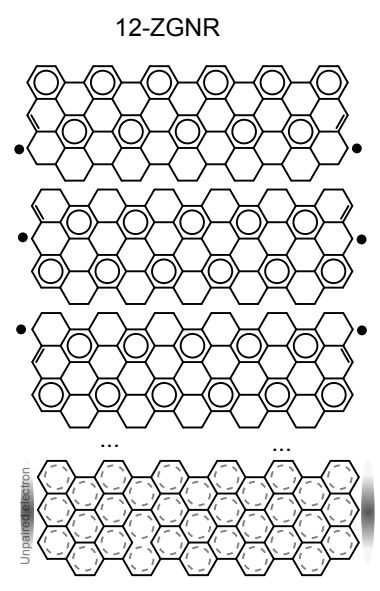

b

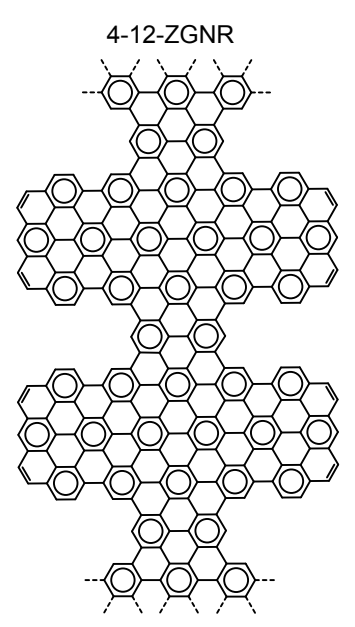

c

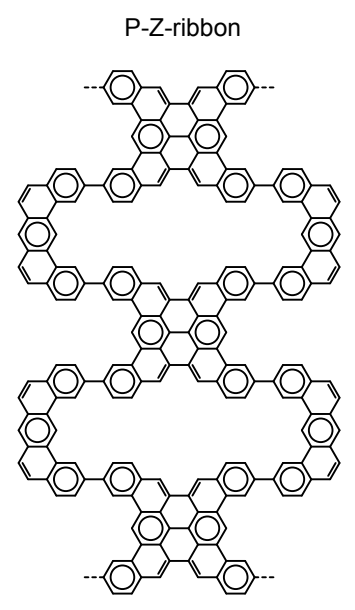

Figure S11: (a) Some typical Clar formulas are shown of 12-ZGNRs, as well as their superposition evidencing delocalized Clar sextets along the ribbon and unpaired electrons along the zigzag edges (b,c) Clar formulas are shown of 4-12-ZGNR and P-Z-ribbon respectively evidencing no unpaired electrons existing along the zigzag edges.

\section{Clar's theory analysis}

. We have analyzed the P-Z-ribbon heterostructure upon Clar's theory ${ }^{2}$ For comparison, the representative Clar formulas of 12-ZGNR and 4-P12-ZGNR are also shown in Fig.S9a,b, with the highest number of Clar sextets pictured as circles within the corresponding carbon hexagons, ${ }^{3}$ which can provide a visual framework to comprehend the electronic and magnetic structures. For 12-ZGNR, the combination of all possible Clar formulas (for the limited layout space only three typical Clar formulas are shown for 12-ZGNR) should show all hexagons are Clar sextets simultaneously with unpaired electrons existing at both zigzag edges, suggesting the origin of spin polarized zigzag edge states. In contrast, there is only one Clar formula with the maximum Clar sextets for both 4-12-ZGNR and P-Z-ribbon heterostructures, which show notable bond length variations within the heterostructure indicating a prospective band gap, and no unpaired electrons existing at the heterostructure edges implying no spin polarized edge states should be expected in these systems. Additionally, as the Lieb's theorem predicted that the total spin of a bipartite system can be determined by the number of atoms on the sublattices of $\mathrm{A}$ and $\mathrm{B},{ }^{4}$ the calculated total spin of P-Z-ribbon 
should also be zero due to the equal numbers of $\mathrm{A}$ and $\mathrm{B}$ sublattice in P-Z-ribbon.

\section{References}

(1) Gutzler, Rico, Fu, Chaoying, Dadvand, Afshin, , MacLeod, Jennifer M, Rosei, Federico, \& Perepichka, Dmitrii F Halogen bonds in 2D supramolecular self-assembly of organic semiconductors. Nanoscale 4, 5965-5971 (2012).

(2) Clar, E. Aromatic sextet (1972).

(3) Wassmann, T., Seitsonen, A. P., Saitta, A. M., Lazzeri, M. \& Mauri, F. Clar's theory, $\pi$-electron distribution, and geometry of graphene nanoribbons. Journal of the American Chemical Society 132, 3440-3451 (2010).

(4) Lieb, E. H. Two theorems on the hubbard model. Physical review letters 62, 1201 (1989). 